\title{
Understanding baffle overloads observed in high-mirror configuration on Wendelstein 7-X
}

Yu Gao ${ }^{1}$, Yuhe Feng ${ }^{1}$, Marcin W. Jakubowski ${ }^{1,2}$, Joachim Geiger ${ }^{1}$, Michael Endler ${ }^{1}$, Chandra Prakash Dhard ${ }^{1}$, Christoph Biedermann ${ }^{1}$, Dirk Naujoks ${ }^{1}$, Thomas Sunn Pedersen $^{1}$, Ralf König ${ }^{1}$, Peter Drewelow ${ }^{1}$, Fabio Pisano ${ }^{3}$, Aleix Puig Sitjes ${ }^{1}$, Holger Niemann $^{1}$, Sergey Bozhenkov ${ }^{1}$, Samuel Lazerson ${ }^{1}$, Matthias Otte ${ }^{1}$, Joris Fellinger ${ }^{1}$, Jiawu Zhu ${ }^{1}$, Maciej Krychowiak ${ }^{1}$, Daihong Zhang ${ }^{1}$, Barbara Cannas ${ }^{3}$, Yasuhiro Suzuki $^{4,5}$, and the W7-X Team ${ }^{1}$

${ }^{1}$ Max-Planck-Institut für Plasmaphysik, 17491 Greifswald, Germany

${ }^{2}$ University of Szczecin, Institute of Physics, 70-453 Szczecin, Poland

${ }^{3}$ University of Cagliari, Department of Electrical and Electronic Engineering, 09123

Cagliari, Italy

${ }^{4}$ National Institute for Fusion Science, National Institutes of Natural Sciences, Toki, Japan

${ }^{5}$ SOKENDAI (The Graduate University for Advanced Studies), Toki, Japan

August 6, 2020

E-mail: yu.gao@ipp.mpg.de

\begin{abstract}
The operational regimes in the first divertor campaign of W7-X were limited by unexpectedly high heat loads on certain baffle tiles. In the high-mirror configuration, a permanent hotspot on the baffle plate was detected by the thermographic system, which was further confirmed during the post-campaign inspections of the plasmafacing components. The maximum heat load on three baffle tiles reached about $4.5 \mathrm{MW} \mathrm{m}^{-2}$, which was almost an order of magnitude above its designed value of $0.5 \mathrm{MW} \mathrm{m}^{-2}$. The paper presents a detailed analysis to understand how the baffle plate, which is originally designed to screen the recycling neutrals and is therefore hidden from the hot plasma, can receive such a high heat load - a level that is expected for target plates. Three main causes have been identified: 1) the three baffle tiles are radially only about $5 \mathrm{~mm}$ away from a main heat channel towards a target, which is much shorter than the radial power decay length of several centimeters derived from the thermographic measurements in this region; 2) the three baffle tiles are intersected with field-lines of about $30 \mathrm{~m}$ length, which are long enough to collect considerable amount of heat via cross-field transport; 3) the baffle tiles have locally large grazing angles. In addition, the analysis method shown in this paper is of general use to estimate the heat flows in shadow areas of targets and thus to evaluate heat loads on the potentially critical components occurring in these areas, especially required for the design of complex 3D divertors like the one in $\mathrm{W} 7-\mathrm{X}$.
\end{abstract}

Keywords: baffle; overload; W7-X; stellarator; divertor; heat loads 


\section{Introduction}

Wendelstein 7-X (W7-X), the world's most advanced stellarator, has successfully completed its first divertor campaign $[1,2,3,4]$ with ten inertially cooled fine-grain graphite test divertor units (TDUs). In typical attached plasma conditions, when major heat loads on the divertor plates stay well below the upper thermal limit, significant overloads of some local baffle tiles were observed in various magnetic configurations. Higher plasma density or strong impurity seeding was required for the suppression of the baffle loads, which at the same time triggered the power detachment from the divertor plate. In the first divertor campaign (OP 1.2), the baffle loads strongly limited the pulse length and constrained the operational window of $\mathrm{W} 7-\mathrm{X}$. The unexpectedly high heat fluxes observed on a few baffle tiles, which are 5 times larger than those predicted by the numerical calculations performed during the design phase, have to be understood. In this paper, one of the most common baffle loads observed throughout the campaign will be analyzed in detail and interpreted with a series of simulations. This work aims to provide additional insight to allow precautions in the general design of plasma-facing components (PFCs) interacting with three dimensionally (3D) shaped plasmas in the future.

The magnetic configuration of W7-X has emerged from an optimization procedure which aimed at curing the well-known deficiencies of classical stellarators to be viable fusion reactor candidates: good flux surfaces, high equilibrium beta-limit, a large enough MHD-stability beta-limit of around $5 \%$ of volume-averaged beta, low neoclassical transport and a small bootstrap current in the long-mean-free-path regime, and a good confinement of fast particles [5]. The point-optimized configuration resulting from the procedure is a compromise with respect to the various criteria. This theoretical configuration with 5 field periods, a rotational transform profile around $t=1$ with a low shear in order not to cross major resonances inside the confinement region, with a major radius of $5.5 \mathrm{~m}$ and a minor radius of $0.5 \mathrm{~m}$, has been realized with a superconducting coil system which was designed to allow for changes in the configurations in order to explore the various optimization targets. Here, the rotational transform $t$ is defined as the number of poloidal turns per single toroidal transit of a field line on a toroidal flux surface. In terms of

tokamaks $t=\frac{1}{q}, q$ being the safety factor. The optimized magnetic topology is realized with this coil system in the so-called high-mirror configuration. The naming illustrates that a toroidal mirror field is the characteristic for this configuration: the magnetic field strength on axis varies in one period by $\pm 10 \%$ from the average field strength. Deviating from this configuration to improve one of the optimization goals will violate the balance of the optimization criteria on the expense of the others. The high-mirror configuration has a boundary rotational transform $t_{a}$ of 1 which allows for the formation of the $n / m=5 / 5$ ( $n, m$ for toroidal and poloidal mode number) islands for an interaction with a divertor for particle and energy exhaust which is named the island-divertor concept. The mirror field of the high-mirror configuration is important for two aspects, for a small bootstrap current and for good fast particle confinement. The small bootstrap current is crucial for stable divertor operation in order to keep the boundary islands at the proper radial location with respect to the divertor plates, otherwise an $t_{a}$ control either with current drive or with dynamic configuration changes is needed. Moreover, results show that in the high-mirror configuration the wall loads generated by neutral beam injection (NBI) are reduced making it to be the best candidate for NBI-operation [6].

The mentioned island-divertor concept used in W7-X was tested for the first time in the predecessor experiment W7-AS [7]. In W7-X, the island divertor proved to be 
quite successful with respect to the particle and energy exhaust and even a stable power and particle detachment could be shown in OP $1.2[8,4]$. According to periodicity and stellarator-symmetry (a flip-symmetry in one period) ten divertor units are distributed toroidally with five in the upper (labelled as 1-5u) and five in the lower part (1-5l) of the machine [9, Figure 1 therein]. Each divertor unit consists of horizontal and vertical targets. In an attached divertor operation phase, the dominant plasma heat loads, originating from the confinement region, stream towards the divertor plates along the open field lines inside the magnetic islands in the scrape-off layer (SOL). Non-axisymmetric heat flux patterns are formed by the interaction of the helical island chains and the divertor plates, and have been observed using the wide-angle infrared cameras $[10,11,12]$. Deviations in the experiment from the ideal patterns can arise from error fields $[13,14]$ present in the vacuum fields generated by the superconducting coil system. These originate from inaccuracies in the fabrication and assembly of the coil system and, although small, can lead to noticeable changes in the low-shear magnetic configuration of W7-X. Dedicated experiments using the additionally available coil systems to correct low order error field [15, 16], the socalled trim coils, as well as the planar coils of the main coil system for adjusting the vacuum $t$ [17] have been performed to approximate the ideal magnetic configuration as good as possible. However, particle drifts lead to an up-down asymmetry in the particle and energy fluxes [18] which is seen in all magnetic configurations. Experimental power distributions have been quantitatively analyzed with new methods [9] developed to cope with 3D heat fluxes. Good consistency with numerical predictions, e.g. diffusive field-line tracing [19] or EMC3-EIRENE [20, 21], has been found with respect to the shape and location of the main strike lines [22, 23, 24].

However, overloads of certain baffle tiles were measured in the standard, the low-iota $\left(t_{a}=5 / 6\right)$ and the high-mirror configuration, which limited the operational regime during the campaign [25]. Figure 1 shows the divertor structures together with the baffle plates which extend the divertor plates poloidally on the inboard and on the outboard side. With respect to the heat load limits of divertor components, it should be noted that the middle section of the horizontal target has the same limits as the baffles. All in all, the baffle components (inner and outer plates as well as the middle divertor part) cover $\sim 3 \mathrm{~m}^{2}$ in each divertor unit. They aim to contain neutral particles close to the target plates and assist neutral compression for high pumping efficiency. The geometry of the baffle plates is designed such that the baffles are shadowed from heat loads by divertor plates for all magnetic configurations. Stationary conductive and convective loads to the baffles should therefore not be present. Nevertheless, plasma radiation or stray radiation from the electron cyclotron resonance heating $(\mathrm{ECRH})$ is expected to deposit a non-negligible power onto the baffle components. An upper limit of the heat flux of $\sim 0.5 \mathrm{MW} \mathrm{m}^{-2}$ is allowed for safe steady-state operation of the baffle tiles using their integrated water cooling [26]. This threshold is determined by the critical temperature of the $\mathrm{CuCrZr}$ structure supporting the baffle tiles, rather than by the sublimation temperature of the carbon surface. Figure 2 shows the structure of a baffle tile in detail. It consists of a graphite tile, a layer of SIGRAFLEX ${ }^{\circledR}$ (specific graphite layer ensuring reliable thermal contact between surfaces), a CuCrZr heat sink and an integrated zigzag cooling pipe. The form of the graphite tile is composed of multiple polygon surfaces resulting in a variety of grazing angles of the field lines on the surfaces of the tiles. 4 TZM screws (TZM is an alloy with $99 \%$ Molybdenum mixed with Titanium and Zirconium) are used to fix the tile, with the screw head lowered by $2.5 \mathrm{~mm}$ below the tile surface. A chamfer structure of the graphite surface with an angle of $45^{\circ}$ has been added around each screw head to avoid 
leading edges. In OP 1.2, the baffle plates, being composed of such tiles, were operated without water flowing in the circuits. To ensure safe operation without water-cooling, a surface temperature limit of $\sim 500{ }^{\circ} \mathrm{C}$ for the baffle tiles was required and monitored by the thermographic systems during each discharge.

This paper focuses on a specific critical baffle overloading observed in typical discharges in the high-mirror configuration. Detailed analysis of the experimental measurements and the simulations are performed to achieve a thorough interpretation of the observations. A complete understanding of the overloading is required before any optimizations are envisaged for these baffle tiles for the up-coming campaigns, which will be operated with higher heating power, longer pulses $\sim 1800 \mathrm{~s}$ and with water-cooled PFCs. Section 2 presents the experimental observations of the overloaded baffle tiles, namely, by the thermographic system during the experimental campaign and by the in-vessel inspection after the campaign. Reasons for the experimentally observed high baffle loads will be discussed in section 3 supported by a set of simulations. Finally, section 4 presents the conclusions and a discussion of potential methods to mitigate the unwanted baffle loads.

\section{Observation of the overloaded baffle tiles}

Non-radiative thermal patterns on baffle components were observed at various locations under different magnetic configurations during the campaign. While most baffle loads can be mitigated together with the main strike lines by higher plasma density or by divertor impurity seeding until the onset of complete detachment of the power loads, such scenarios would limit the operational windows for various experimental explorations, especially transport studies requiring relatively low plasma density. This paper focuses on the understanding of one of the most common baffle loads exceeding the upper thermal limits, i.e. the overloads of the inner baffle tiles in the high-mirror configuration.

Figure 3 shows the plasma parameters of discharge 20181009_009 (discharge number at W7-X: DATE_< discharge count of that day $>$ ) which was run in the high-mirror configuration. This discharge had constant plasma parameters over $10 \mathrm{~s}$ with $3 \mathrm{MW}$ of ECRH heating $\left(P_{\mathrm{ECRH}}\right), 0.4 \mathrm{MW}$ of total plasma radiation $\left(P_{\mathrm{rad}}\right)$, a line integrated electron density $\left(\int n_{\mathrm{e}} d l\right)$ of $5 \times 10^{19} \mathrm{~m}^{-2}[27]$ and $400 \mathrm{~kJ}$ diamagnetic energy $\left(W_{\mathrm{dia}}\right)$. Within $10 \mathrm{~s}$ the maximum surface temperature of the critical baffle tile in module 3 lower divertor (3l), whose location is marked in red in figure 1 , increases from $140^{\circ} \mathrm{C}$ to $510^{\circ} \mathrm{C}$, while the average surface temperature of this tile rises from $115^{\circ} \mathrm{C}$ to $285^{\circ} \mathrm{C}$. The measurement assumes a constant surface emissivity of 0.82 , which was calibrated for the graphite material used in W7-X. Possible effects of surface layers built from multiple-elements by deposition on the measured surface temperature and on the associated heat flux calculations are neglected in this paper.

A thermographic image overlaid on the picture of the CAD components captured at the end of the discharge $(t=10 \mathrm{~s})$ by one of the immersion tube infrared cameras [10] monitoring module $3 \mathrm{l}$ is presented in figure 4 . The temperature patterns on the vertical and horizontal targets reproduce typical thermal footprints predicted for the high-mirror configuration by diffusive field-line tracing simulations, as shown in [1, Figure 5 therein]. However, the temperature pattern in figure 4 also shows rather high temperatures on baffle tiles highlighted by a white contour where the surface temperature reaches a value as high as in the location of the main strike line on the vertical target. Such high baffle loads in the high-mirror configuration were observed throughout the campaign. Dedicated scans of the planar coil currents for modifying $t$ have shown that the baffle loads are persistent but can 
be controlled with a minimum heat load, when the $t_{a}$ of the real magnetic configuration is corrected to the $t_{a}$ of the designed (ideal) high-mirror configuration. In cases with uncorrected $t_{a}$, intolerable baffle loads exceeding even the level of the main strike line on the vertical targets have been observed. For the discharge analyzed here, not only the $t_{a}$ been adjusted correctly [17], but also the best target heat load symmetrization was achieved, using the additional coil system, i.e. the trim coils for compensating the $n=1$ component of the error field and the control coils to additionally compensate also the $n=2$ component $[15,16]$. This specific configuration of coil currents has been determined based on a set of phase and amplitude scans with the trim coils as well as with the control coils beforehand, with the aim to best symmetrize the power distribution among divertor targets in different modules. The properly adjusted $t$ together with the error-field correction allows the vacuum field used in this discharge to be as close as possible to the ideal high-mirror configuration.

For quantitative analysis and comparison, the thermal images from different modules have been restored from the optical distortions caused by the fish-eye lenses used [12], and have been projected onto an identical 2D plane [9]. Heat fluxes have been calculated using the THEODOR code [28] which solves the 2D heat diffusion equation with an explicit method. Considering the asymmetry of power loads among the TDUs due to the remaining error fields, heat fluxes averaged across all machine modules would be preferable to perform the analysis. However, the infrared camera monitoring the lower divertor in module 5 is not available for this discharge. Moreover, to ensure that particle drifts (up-down asymmetries) do not bias the averaged results, we only consider TDUs in pairs (equal number for lower and upper targets). Thus we use an average consisting of only the machine modules 1 to 4 .

The result of the averaging process is shown in figure 5. The upper row shows divertor parts (horizontal and vertical targets and inboard-side baffle) relevant for the high-mirror configuration, while the lower row shows zoom-ins of the highly loaded baffle regions to point out the different thermal footprints of the baffle loads. The middle part of the figure (subfigure (c) and (d)) shows the average over all 8 TDU-modules. In order to show the effect of particle drifts on the up-down asymmetry of the power load distribution which has been investigated the first time in [18], also the results of averaging only the lower (subfigure (a) and (b)) and only the upper TDUs (subfigure (e) and (f)) are shown. Strike line patterns on the horizontal and vertical target plates remain narrow and clear after averaging, little affected by the rather small differences of the strike line locations among different TDUs. Particle drifts seem not to change the main strike line locations for high-mirror configuration but rather modify the amplitude of the heat loads, e.g. the upper vertical targets receive more heat load than the lower vertical targets, while for the horizontal target the opposite is the case. Two subsections are defined as baffle left and baffle right within the set of 8 loaded baffle tiles. Here, baffle left refers to the baffle area receiving plasma heat loads from the low-iota end (defined in figure 1), while baffle right refers to the baffle area receiving plasma heat loads towards the low-iota end. The complicated structure of the watershed on the inner baffle plate will be described in section 3. Quantitative comparisons have been made between the different averaging cases, i.e. averaging over only the lower TDUs, the upper TDUs and all TDUs (with the above restriction to modules 1 to 4 ), which are shown in table 1 . The average heat fluxes of the different regions have been normalized to the case using all vertical TDU targets. Note that for the averaged heat fluxes the lower TDU has a higher ratio between the baffle left and baffle right $\sim \frac{0.485}{0.501}=0.968$ than the upper TDU $\sim \frac{0.416}{0.718}=0.579$. Such 
experimental results provide direct evidence that the observed baffle loads have certain contributions from convective losses of heat and particles, which are connected to particle drifts.

\begin{tabular}{|c||c|c|c|}
\hline \multicolumn{4}{|c|}{ Normalized averaged heat flux } \\
\hline & lower TDUs & all TDUs & upper TDUs \\
\hline Vertical target & 0.903 & 1 & 1.097 \\
Baffle left & 0.485 & 0.450 & 0.416 \\
Baffle right & 0.501 & 0.610 & 0.718 \\
\hline
\end{tabular}

Table 1: Averaged heat flux on different areas (normalized to the case of all-TDUsaveraged heat flux on the vertical target). Baffle left and Baffle right are areas defined based on the watershed.

In the all-TDUs-averaged case, the most critical baffle tile, which has been marked in figure 1, is of greatest interest, since an extremely strong hot spot with a peak heat flux of $\sim 1.2 \mathrm{MW} \mathrm{m}^{-2}$ can be seen in the region of the bottom right screw, while the major area of the tile receives heat loads of only $\sim 0.4 \mathrm{MW} \mathrm{m}^{-2}$. This hot spot appears to be the peak heat flux location on the whole baffle plate for most TDUs. Figure 5 shows a comparison of the average and peak heat flux of the eight most loaded baffle tiles for the different TDU modules. Peak baffle loads exceeding the upper limit of $0.5 \mathrm{MW} \mathrm{m}^{-2}$ can be observed for all TDUs, with $3 \mathrm{u}$ receiving the highest baffle loads with a peak heat flux of $\sim 4.6 \mathrm{MW} \mathrm{m}^{-2}$. The locations of the highest heat load are almost identical for all TDUs, represented by a hot spot in the vicinity of the screw region as indicated in the averaged heat flux map.

The observed hot spots during the experiments have been carefully checked after the campaign, during the in-vessel inspections. The critical area has been identified not to be on the screw head, but on the chamfer structure surrounding the screw as shown in figure 7 . The main picture is from the TDU module $5 \mathrm{u}$ and covers a part of the vertical target as well as a part of the inner baffle plate. The zoom-in picture on the top right corner shows the critical screw region in more detail. Direct interactions with particles at the chamfer structure are confirmed by a rather rough and bright pattern visible at the left part of the chamfer. Since the pattern only exists on the left part, it is considered to be due to erosion caused by particles flowing toroidally towards the low-iota end. Postmortem analysis has to be done to determine the depth of the erosion, considering the potential deposition layers around it. Multiple scratches can also be seen on the surface of the graphite tile to the left of the screw. The formation of these scratches is not clear, but it seems that they all point in the direction perpendicular to the magnetic field. There are several possibilities to speculate on the origin of the scratches including electric arcs of plasma causing 'magnetized' [29] patterns in $-\mathbf{J} \times \mathbf{B}$ direction, field-aligned filaments drifting inside the island in $\mathbf{E} \times \mathbf{B}$ direction [30] or damages already done before the campaign possibly during manufacturing or assembly. Colorful traces are also observed surrounding the screw, which are considered to be deposition layers consisting of different impurities. Such traces are also seen on the vertical target extending towards the upper boundary of the target, which is consistent with the upper contour of the experimental strike line observed in figure 5. However, the observed deposition layers are attributed to impurity transport throughout the whole campaign, and may largely be influenced or modified by the last-day operation conducting identical discharges in the standard magnetic configuration with active ${ }^{13} \mathrm{C}$ tracer particle injections. It should be noted that 
the erosion patterns at the chamfer corner have been found also in some other modules at respectively identical locations, but with different levels of severity.

\section{Physical interpretation}

Heat and particle exhaust in W7-X is complicated due to the 3D geometry of the helical island chains in the SOL and the correspondingly shaped divertor geometry. Discrete divertor plates are locally curved (as shown in figure 1) to intersect the island chains concentrating the plasma-wall-interaction to hardened targets in order to prevent the hot plasma from damaging other in-vessel components with lower thermal limits, e.g. wall and baffle. The divertor shape has been optimized to be compatible with the flexibility in the space of magnetic configurations. In tokamaks, opposite plasma flows are generally separated far away from each other and guided by the two legs from the X-point to the so called outer and inner strike points. In W7-X, depending on the island geometry, opposite flow channels can be adjacent to each other within one target plate, either poloidally [31, Figure 2 therein], or radially because of shadowing effect of the divertor [32, Figure 4 therein]. Going along toroidally on a divertor plate, the flow directions may also change because the grazing angle of flux tubes intersecting the divertor plate changes sign. Such lines where the grazing angle changes sign are called Watersheds and can extend poloidally or toroidally [33, Figure 6 therein] [34, Figure 2 therein] and depend mainly on the magnetic configuration but are modified by plasma $\beta$ and toroidal plasma current. Since the heat transport, guided by the field lines, directs always towards the PFCs, a watershed naturally forms a separation belt for plasma flows in the opposite toroidal directions. Here, the terms "opposite" or "reversed flow" are introduced only to illustrate the fact that the plasma (particles and heat) flows in two different directions parallel to the magnetic field to different components or to certain areas of them, where, according to the Bohm condition [35], the particles reach the ion sound speed and each carries a certain amount of energy to a solid surface. There is no implication of any transport processes on the way along a flux tube to a solid surface.

The baffle tiles with high heat loads discussed in this paper are located in the vicinity of the watershed. As shown in figure 8 (a), for the high-mirror configuration a watershed on the vertical target stretches poloidally from the toroidal angle of $\sim-15^{\circ}$ to $\sim-16^{\circ}$, and extends to the baffle plate. Due to the complex polygonal shaping of the baffle tile, the watershed is rather irregular on the baffle plate depending on the local geometry of the tile surface. The position with toroidal angle of $-15.5^{\circ}$ is used as an approximation of the watershed line in high-mirror configuration.

The major heat loads onto the baffle plate seen in the experiments are coming from plasma flowing towards the low-iota end, while a relatively small amount of heat flux is deposited by the opposite flow as inferred from combining the information from figure 8 (a), figure 5 and table 1 . Note the details of the heat load patterns of the upper and lower baffle tiles in figure 5 (b) and (f) are different and depend on the locally distributed watersheds on the different tiles. The upper baffle tiles generally receive more heat loads flowing towards the low-iota end, while the lower baffle tiles receive more from the low-iota end, when operating in the positive field direction, which has the toroidal field component in the positive $\phi$ direction as marked in figure 1 . The enhancement or reduction of particles flowing in either direction by particle drift is observed but the detailed understanding of the effects is out of the scope of this paper (for more see [18]).

One reason for the extremely localized heat loads on the baffle is the rather large 
grazing angles of the polygon surfaces on the baffle tiles. Figure 8 (b) shows a strongly nonuniform distribution of the grazing angle, even over one tile surface. A large grazing angle $\alpha>12^{\circ}$ is measured on the critical baffle tiles at the plasma-facing side. The heat flux perpendicular to the baffle tile is amplified by a specific grazing angle as $q_{\perp, \text { surface }}=q_{\|}$sin $\alpha$, where $q_{\|}$is the parallel heat flux along the field line.

A series of simulations has been performed for the high-mirror configuration to understand why significant power could be deposited onto the baffle plate, which by design ought to be shaded from parallel heat flux by the target plates. Firstly, the wall to wall connection length $\left(L_{\mathrm{c}}\right)$ of the field lines intersecting the baffle and target plates are calculated via field-line tracing as shown in figure 9 (a). In order to highlight the situation of the inner baffle, the scale of the colorbar is limited to $40 \mathrm{~m}$, while the main strike line on the vertical target actually has a maximum $L_{\mathrm{c}}$ of $\sim 1 \mathrm{~km}$. The dark red areas on the vertical and horizontal target plates are the regions that are connected with field lines with $L_{\mathrm{c}}$ of several 100 meters. These long field lines form the main parallel heat channels through which the power entering the islands at the core periphery is transported onto the targets. There are five such channels in total, corresponding to the poloidal number of the islands, each connecting two divertor units. In general, the field lines touching the inner baffle plate have much shorter $L_{\mathrm{c}}$, meaning that the baffle plate is indeed in the shadow of the target plates. However, while most baffle tiles show a typical $L_{\mathrm{c}}$ of $3.5 \mathrm{~m}$, three of them are intersecting with much longer field lines up to $32 \mathrm{~m}$. These three tiles are also the most heavily loaded ones observed in experiments within the defined baffle right area. Thus, there must be a close correlation between the baffle overload and the exceptionally long flux tube on the baffle plate.

The identification of the intersection of the three baffle tiles with a relatively long flux tube is, however, insufficient for explaining the observed baffle load. As a prerequisite for a heat flux in the flux tube, the flux tube must be able to collect enough heat from somewhere. Having a $L_{\mathrm{c}}$ of $32 \mathrm{~m}$, this flux tube is radially remote from the core boundary where the heat comes from, but is adjacent to a main parallel heat channel. In fact, this flux tube is an extended part of the flux tube in contact with a main parallel heat channel, which can be clearly seen from its map on the vertical target and baffle plate, as shown by the orange contour in figure 9 (a). Figure 9 (b) shows the $L_{\mathrm{c}}$ distributions in real space at the toroidal angle of $-15^{\circ}$, which is at the center of the most critical baffle tile. The colorbar in the zoom-in figure is adjusted in order to show both the $L_{\mathrm{c}}=32 \mathrm{~m}$ and $35.5 \mathrm{~m}$ region. The tile intersects first with a flux tube of $L_{\mathrm{c}}=32 \mathrm{~m}$, which is radially adjacent to a $35.5 \mathrm{~m}$ channel and then the main parallel heat channel with $L_{\mathrm{c}}>500 \mathrm{~m}$. In fact, the tile is geometrically protected by layers of flux tubes from parallel transport of hot plasma from the separatrix, but the radial distance between them is extremely short with only $5.5 \mathrm{~mm}$. Similar radial closeness to the main heat flux channel is observed for all the three baffle tiles with $L_{\mathrm{c}}$ of $32 \mathrm{~m}$. It is reasonable to expect that, through cross-field transport processes, a certain amount of heat can be transferred from the main channel into the adjacent flux tube terminating on the three critical baffle tiles. Besides the three most critical baffle tiles, their neighbors on the low-iota side (see figure 1 for definition) receive less but also considerable heat fluxes as shown in table 1 (baffle left). In fact, these tiles are covered by another flux tube of a much shorter $L_{\mathrm{c}}$ of $3.5 \mathrm{~m}$, which is radially adjacent via the same $35.5 \mathrm{~m}$ channel to the main parallel heat channel with $L_{\mathrm{c}}>500 \mathrm{~m}$, too. Such narrow sheaths can reduce but not eliminate power load transfers to the baffle tiles by cross-field transport from the main heat channel.

Figure 10 shows the trajectories of two representative field lines starting from the inner 
baffle plate (red for baffle right, green for baffle left) in module 3l. Both are terminating at the neighboring upper vertical target, but coming from different toroidal directions with respect to the watershed as explained in figure 8 (a). Given the same conditions, a longer flux tube should gain more heat than a shorter one, just in view of its longer collection length [36, Figure 11 therein]. A longer $L_{\mathrm{c}}$ gives the flux tube a larger area over which to collect heat and particles from the adjacent field lines that are closer to the last closed flux surfaces. This is indeed supported qualitatively by experimental observations presented in section 2 that the baffle right region receives generally more heat load than the baffle left one. The thin $L_{\mathrm{c}}=35.5 \mathrm{~m}$ flux tube layer shown in figure 9 (b) is actually the combination of the green and red field lines without the touching points on the 31 baffle plate. This flux tube is shown as a black line in figure 10. In other words, plasma entering radially the $35.5 \mathrm{~m}$ flux tube from the core would pass over the baffle tiles and deposit the power directly to the vertical target ( $2 u$ in this case) at either side of the watershed, if only parallel transport is considered. This means that the vertical target is the only component for shading the baffle tiles discussed here.

The end points in $2 \mathrm{u}$ are mapped via stellarator-symmetric technique back to 31 to see where the flux tube connects with respect to the main heat channel in figure 10. This shows that the longer field line (red) connects the most critically loaded baffle tile to the tail of the experimentally observed main strike line, while the short field line (green) ends at the boundary of the main heat channel close to the low-iota end. The same technique has been applied to the entire area on the baffle plate with $L_{\mathrm{c}}=32 \mathrm{~m}$. The results of this mapping are projected to the 2D plane of the components for a better visualization, and are indicated with white dashed lines in figure 9 (a). The most loaded baffle tiles actually connect to an area on the vertical target with an irregular shape (also indicated with a white dashed line) almost aligned toroidally with the upper boundary of the predicted parallel flow channel (dark red region). In addition, grey dashed lines indicate the two areas connected by the $35.5 \mathrm{~m}$ flux tube, which reside at either side of the watershed on the same vertical target. The part of the flux tube on the right side of the watershed acts like a thin separation belt between the $32 \mathrm{~m}$ flux tube and the main heat channel, consistent with figure 9 (b).

As a summary, figure 11 illustrates the heat transport causing the observed baffle loads. Here, the toroidal angle $0^{\circ}$ (different from the angle in the real $\mathrm{W} 7-\mathrm{X}$ device) is defined as the location of the watershed on the vertical target, indicated by the black dashed line in figure 10. In the high-mirror configuration, the predominant parallel transport channel deposits the power loads to the vertical target. The baffle plate is indeed shaded from this direct transport channel, but the critical baffle tiles are only $5.5 \mathrm{~mm}$ radially away from it. By cross-field transport, power can first enter the $35.5 \mathrm{~m}$ flux tube connecting either side of the same target, and then enter further outwards the $32 \mathrm{~m}$ and $3.5 \mathrm{~m}$ flux tubes reaching the baffle plate from opposite directions. Note that the overload on the baffle plate is caused by the heat flux along the $32 \mathrm{~m}$ flux tube which is in the opposite direction compared to the direction of the main heat channel.

Experiments confirm that bidirectional flows exist within the $L_{\mathrm{c}}=32 \mathrm{~m}$ flux tube but with different level of heat flux to each end. In figure 5, the regions connected by this flux tube have been marked with white dashed lines. Combining simulations and experiment, one can actually calculate the parallel heat flux $q_{\|}$in both directions for the $32 \mathrm{~m}$ flux tube. While the average power load to the baffle plate $\overline{q_{\|}^{\mathrm{b}}}$ is $4.2 \mathrm{MW} \mathrm{m}^{-2}$, it is much larger for the vertical target $\overline{q_{\|}^{t}}=9.4 \mathrm{MW} \mathrm{m}^{-2}$. From this a ratio of 2.2 between the parallel heat flux flowing to the vertical target and the one flowing to the baffle plate 
is determined. Such asymmetry is reasonable because the origin of the heat flux entering this flux tube is from the main parallel heat channel, where particles predominantly flow to the vertical target as shown in figure 11. More specifically, it is very close to the end of the main channel, i.e. $\sim 32 \mathrm{~m}$ before reaching the vertical target, where the ions are accelerated towards sound speed. On the one hand, plasma initially diffusing into this flux tube would carry its original momentum being mainly towards the target. On the other hand, considering the momentum exchange by particle collision and friction, a higher $q_{\|}$ is expected at the target end of the flux tube rather than at the baffle end [32, Figure 4 therein].

The schematic of figure 11 tells that the cross-field transport must have a characteristic scale-length larger than $5.5 \mathrm{~mm}$ to cause such baffle loads. To estimate the perpendicular transport in detail using the existing experimental results, the intersection of the flux tube has been mapped together with the experimental heat flux on the vertical target to a specific poloidal cross-section at the upstream position by a backward field-line tracing. Figure 12 shows the result in the poloidal cross-section at the toroidal angle of $0^{\circ}$ (location indicated in figure 1). The area in figure 12 which is marked with a white dotted line (flux tube region with $L_{\mathrm{c}}=32 \mathrm{~m}$ ) covers a region with strong $q_{\|}$, which suggests that some power must have accessed this flux tube through spreading of parallel heat channel by cross-field transport.

For a more quantitative analysis, heat flux profiles along a line on the vertical target at toroidal angle of $-7.7^{\circ}$ (marked with purple dashed line in figure 9 (a)) are extracted to compare on the one hand with the surface $L_{\mathrm{c}}$ and on the other hand with heat fluxes from simulations with the SOL transport code EMC3-EIRENE. First, figure 13 (a) shows selected experimentally determined heat flux profiles from different TDUs. Clear differences can be seen in the profiles, which are mainly due to the remaining error fields, particle drifts and a supposed finite misalignment of the different divertor modules [34]. The peak heat fluxes range from $0.9 \mathrm{MW} \mathrm{m}^{-2}$ to $1.8 \mathrm{MW} \mathrm{m}^{-2}$, while the peaking locations have a maximum displacement of $\sim 6 \mathrm{~cm}$. To compare with simulations, averaging of these profiles is necessary in order to suppress these effects for simplicity since they are so far not included in the modelling. For this purpose we apply two averaging methods. The first one averages directly the profiles using the fixed target coordinate, with the result shown in yellow dots in figure 13 (b). The second method first aligns each profile with respect to its peak location and then perform the average. This one is shown as black-dotted curve. The two methods result in very similar profiles, with only a slightly higher peak heat flux in the case of prior peak-alignment. Little differences between the different averaging methods are found for the estimation of power decay in the $L_{\mathrm{c}}=32 \mathrm{~m}$ flux tube region.

Figure 13 (b) also shows the $L_{\mathrm{c}}$ profile. This has a sudden cut off at location of $\sim 18 \mathrm{~cm}$ away from the pumping gap, where $L_{\mathrm{c}}$ drops rapidly from $\sim 470 \mathrm{~m}$ to $\sim 35.5 \mathrm{~m}$. The area in purple shows the range on the vertical target where the $32 \mathrm{~m}$ long flux tube connects to the baffle plate on the other end. The cut off of the $L_{\mathrm{c}}$ profile left to the purple area confirms that no power from the main heat channel can reach this flux tube via parallel heat transport.

In contrast to the $L_{\mathrm{c}}$ profile, the experimentally averaged heat flux profile has a smooth Gaussian-like shape and decays continuously from $\sim 16 \mathrm{~cm}$ until $\sim 26 \mathrm{~cm}$ away from the pumping gap (power decay length of $\sim 10 \mathrm{~cm}$ ), which obviously covering the region of the flux tube. Such heat flux broadening by perpendicular transport is observed in all fusion devices, but it is specifically enhanced in W7-X due to the long $L_{\mathrm{c}}$, which is ten 
times larger than that in medium sized tokamaks [37]. Usually, in tokamaks the integral power width [38] is used to characterize the width of the heat flux profile, which is defined here as the integral of the heat flux along the vertical target divided by its peak heat flux $\int q_{\text {vertical }}(r) d r / q_{\text {vertical }}^{\max }$. For the experimental heat flux profile averaged using the fixed target coordinate, the integral power width is calculated to be $9.1 \mathrm{~cm}$. In fact, the left side of the flux tube is located at around one third of the power decay length, i.e. $3 \mathrm{~cm}$ away from the peak, which has the highest gradient of the decay. Such a narrow distance, much smaller than the decay length, is the reason for the significant provision of heat and particles into this flux tube.

For a qualitative understanding of the effects of plasma parameters on the power decay length, EMC3-EIRENE simulations have been performed and the results are shown in figure 13 (b), too. Three different scenarios are simulated with combining two different perpendicular particle diffusivities $(D)$ and two separatrix electron densities $\left(n_{\mathrm{e}}\right)$. In all three cases, a constant heating power of $3 \mathrm{MW}$ is applied assuming no radiative dissipation, which is similar to the discharge conditions. The perpendicular heat transport coefficient $\chi$ is set as $\chi=3 D$. In general, a reduction of the peak heat flux is observed when $n_{\mathrm{e}}$ or $D$ is increased in the simulation. Especially when $D$ is doubled, the peak heat flux is reduced by $\sim 50 \%$ (comparing the light blue line with the green line). Perfect match with the experimental result is not possible due to the lack of accuracy in the measurement for the seperatrix $n_{\mathrm{e}}$. However, simulations indicate that the variations in the input parameters do not change the integral power entering the $L_{\mathrm{c}}=32 \mathrm{~m}$ flux tube region. The flux tube region always covers about the last two thirds of the power decay length for all the simulated heat flux profiles and as a result it receives considerable amount of power, which is consistent with the experimental profiles.

Diffusive field line tracing [19] is widely used as an efficient tool to simulate the thermal footprints of the main strike lines. However, this tool in the current state can not be applied to estimate the baffle loads discussed in this paper, because the reversed flow in the flux tube demonstrated in figure 11 causing the overloads on the baffle tiles does not exist in the model. The code has no boundary conditions at the PFCs, which is normally governed by the Bohm sheath criterion. A single flow direction (forward or reverse with respect to the magnetic field vector) for particles is pre-defined throughout the entire tracing process from the upstream location until the trace intersects with PFCs, without the possibility to reverse the tracing direction. Because of the absence of such backwards parallel flows, the baffle loads produced from this code are five times less than the experimentally observed ones. Most simulated particles carrying the heat are flowing to one end of the flux tube, i.e. the vertical target, and can only reach the critical baffle by large perpendicular steps directly from the main heat channel. The lack of this physics process in the code inhibits this tool to properly simulate the discussed baffle loads. An implementation of a Monte-Carlo flow reversal at each tracing step is foreseen in the future as a first step in the further development of this code package to mitigate this deficiency.

Figure 14 shows the heat flux map produced with the EMC3-EIRENE code, which has a Bohm boundary condition. The result is generated with input parameters of $3 \mathrm{MW}$ heating power, separatrix $n_{\mathrm{e}}=0.5 \times 10^{19} \mathrm{~m}^{-3}$, and $D=1 \mathrm{~m}^{2} \mathrm{~s}^{-1}$. While the main strike line reproduces the experimental results, the baffle loads are also consistent with the measurements, with respect to the power distributions and their amplitudes. The ratio between baffle right and baffle left separated by the watershed is well demonstrated in the simulation being similar to the experimental results shown in figure 5 and table 1 . The heat flux on the critical baffle tiles reach a value of $\sim 0.7 \mathrm{MW} \mathrm{m}^{-2}$, which is also 
of the same level as in the experiment. The experimental hot spots seen at the screw region, however, can not be reproduced because the fine polygon structures of the baffle tiles are not included in the component model used in the simulation. On the other hand, the maximum heat flux at the strike line on the vertical target derived from the infrared image is overemphasized by the leading edge [34] of the protruding graphite tile. Except for these technical issues, an absolute agreement between the simulated and the experimental heat load patterns is not expected due to the incompleteness of the physical model adopted in EMC3-EIRENE. Nevertheless, neither the reduced grid resolution nor the use of simplified PFCs in the 3D code changes the conclusion that overloads on the baffle tiles are consistent with the presented EMC3-EIRENE simulation.

\section{Conclusion and discussion}

Overloads of baffle tiles at the inner baffle plate in all TDUs have been measured by infrared diagnostics in the high-mirror configuration with the best $t$-adjustment and error field correction. Hot spots are observed from the thermal footprints of the loaded baffle tiles, where erosion is found at one side of the chamfer structure of the screw on the most critical baffle tiles during the in-vessel inspection. Multiple scratches are found close to the eroded chamfer in the direction perpendicular to the magnetic field line, suggesting possible formation of electric arcs or strong convective losses at this location.

Simulations indicate that the overloaded baffle tiles shaded from parallel transport by the vertical target are nevertheless vulnerable to perpendicular transport and receive an unacceptable heat flux due to the close radial proximity $(\sim 5.5 \mathrm{~mm})$ of the hot separatrix plasma, relatively long values of $L_{\mathrm{c}}(\sim 32 \mathrm{~m})$ and large grazing angles $\alpha>12^{\circ}$ between the magnetic field line and the baffle surface. The critical baffle tile is located near the watershed, where plasmas flow towards the components from opposite directions. A dependence of the flow directions on the general up-down asymmetry of the power distributions in the high-mirror configuration is found, which also affects the fraction of baffle loads on each side of the watershed. A flux tube connecting the critical baffle tiles resides within the scale of the power decay length of the main heat channel. While the major flow direction within this flux tube is towards the vertical target, the reverse flow fed by cross-field transport is large enough to cause the overloads on the critical baffle tiles.

For the baffle loads simulations in our case, simple diffusive field line tracing is not sufficient, because it lacks the physics of reversed parallel flow. EMC3-EIRENE has been performed with different plasma densities and cross-field transport coefficients. The simulated baffle loads are found to be persistent, with the flux tube carrying the reverse parallel flow accounting for the last two thirds of the power decay length. Quantitative agreement between the simulated heat flux on the baffle plate and the experimental observations is achieved.

Possible solutions to reduce the baffle loads are under investigation in preparation of the future campaigns with water-cooled components, high-power and long-pulse operation. The investigations include magnetic configuration changes applying radial outward shifts of the plasma column for which the planar coils can be used and artificially moving different PFCs towards or away from the plasma. While most approaches do only marginally change the baffle loads, one key parameter found to modify the baffle loads is the relative distance between the vertical target and the critical baffle tiles, as shown in figure 11. In other words, a better shading by the vertical target is required to reduce the 


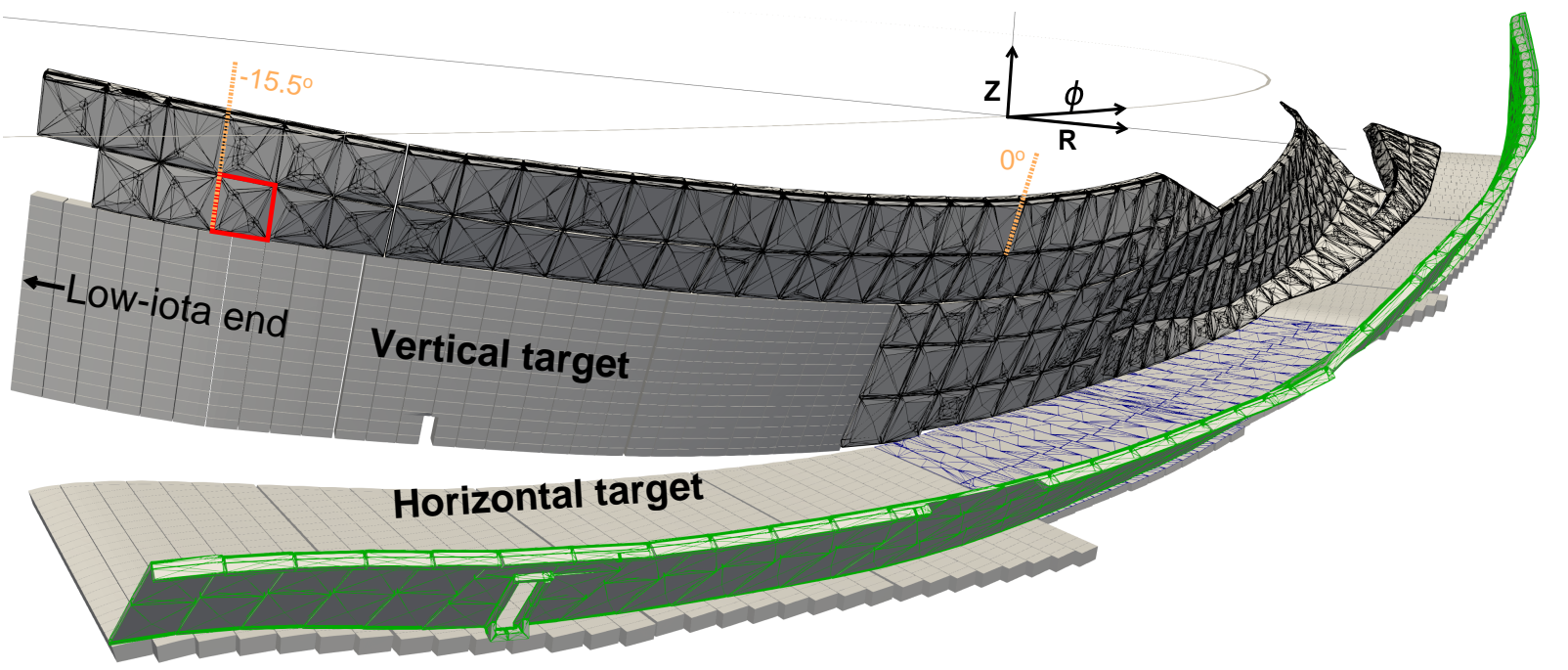

Figure 1: Overview of inner (in black), outer (in green) and middle divertor (in blue) baffle plates installed on $\mathrm{W} 7-\mathrm{X}$ in one divertor unit. The baffle tile enclosed in red is the most critical tile discussed in this paper. The black arrow defines the so-called low-iota end in the TDU. The orange dashed lines indicate the locations of the toroidal angles $-15.5^{\circ}$ and $0^{\circ}$ on the baffle plate structures.

baffle loads. The design of new baffle tiles using tungsten allowing for a reduced thickness $(\sim 4.5 \mathrm{~mm}$ instead of $\sim 10 \mathrm{~mm})$ has been started to replace the 6 most loaded baffle tiles in each divertor module for the up-coming campaign.

\section{Acknowledgments}

This work has been carried out within the framework of the EUROfusion Consortium and has received funding from the Euratom research and training programme 2014-2018 and 2019-2020 under grant agreement No 633053. The views and opinions expressed herein do not necessarily reflect those of the European Commission.

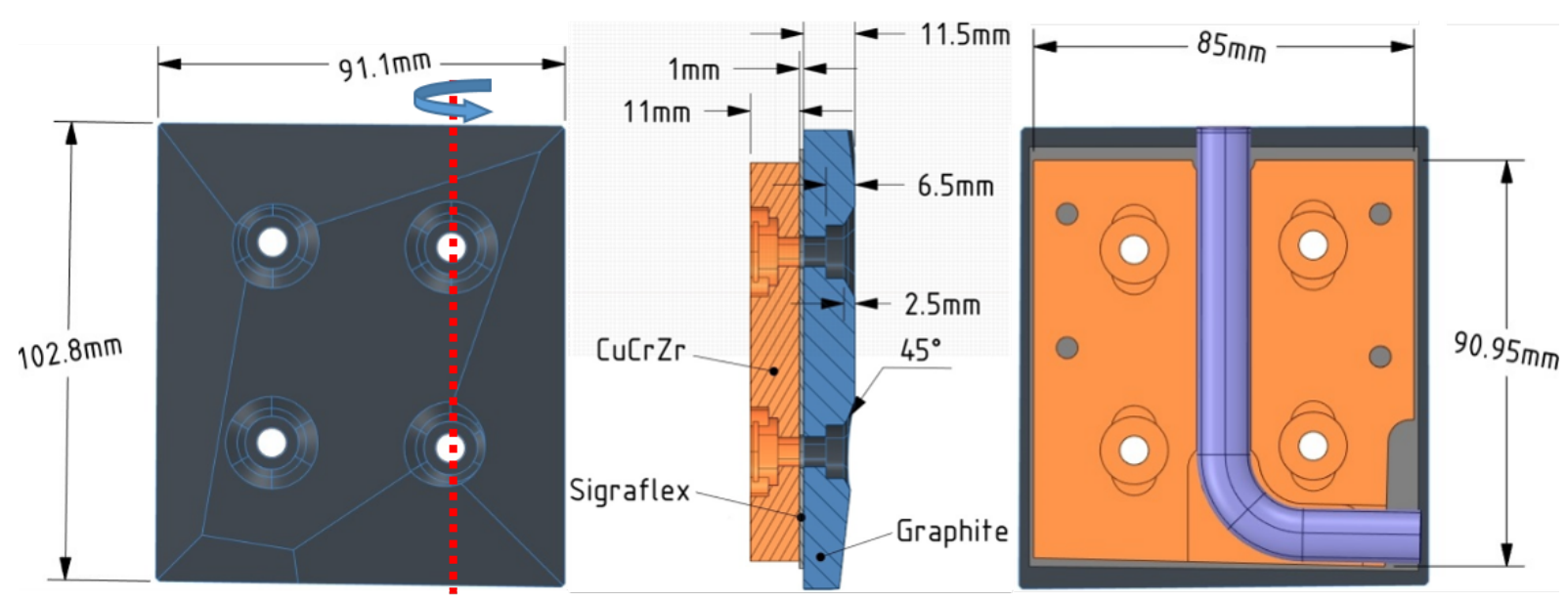

Figure 2: The CAD structure of the critical baffle tile, which is marked in red in figure 1. 


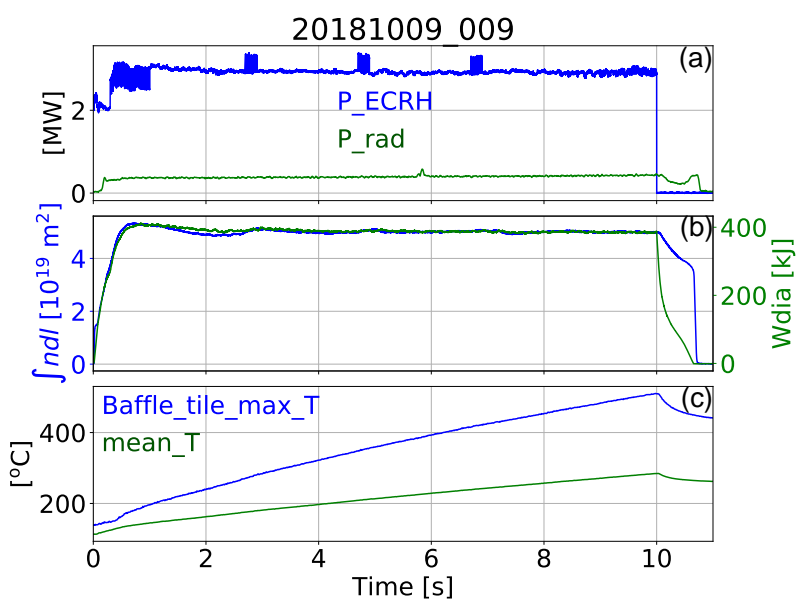

Figure 3: Overview of parameters of the experimental program 20181009_009 in the highmirror configuration. (a) ECRH heating power $P_{\mathrm{ECRH}}$ in blue and total plasma radiation $P_{\text {rad }}$ measured by bolometer in green. (b) The line integrated electron density $\int n_{\mathrm{e}} d l$ measured by interferometer in blue and diamagnetic energy by diamagnetic loop in green. (c) The maximum (in blue) and the average (in green) temperature of the critical baffle tile in module 3 lower divertor (marked in red in figure 1).

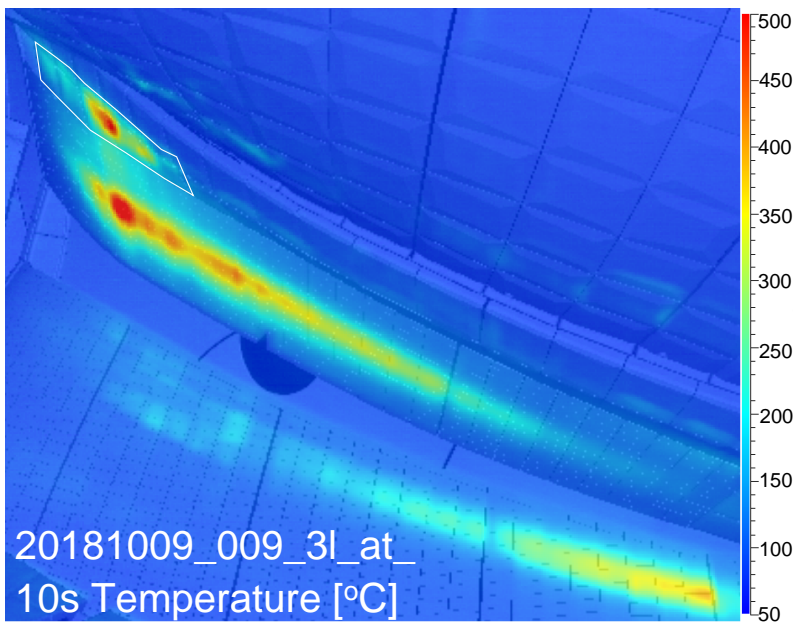

Figure 4: Surface temperature in discharge 20181009_009 at $10 \mathrm{~s}$ measured by the infrared camera mounted on the immersion tube monitoring module 31 . The region of loaded baffle tiles is marked with a white contour. The thermographic picture has been overlaid on the image of the CAD components mapped to the camera view. 

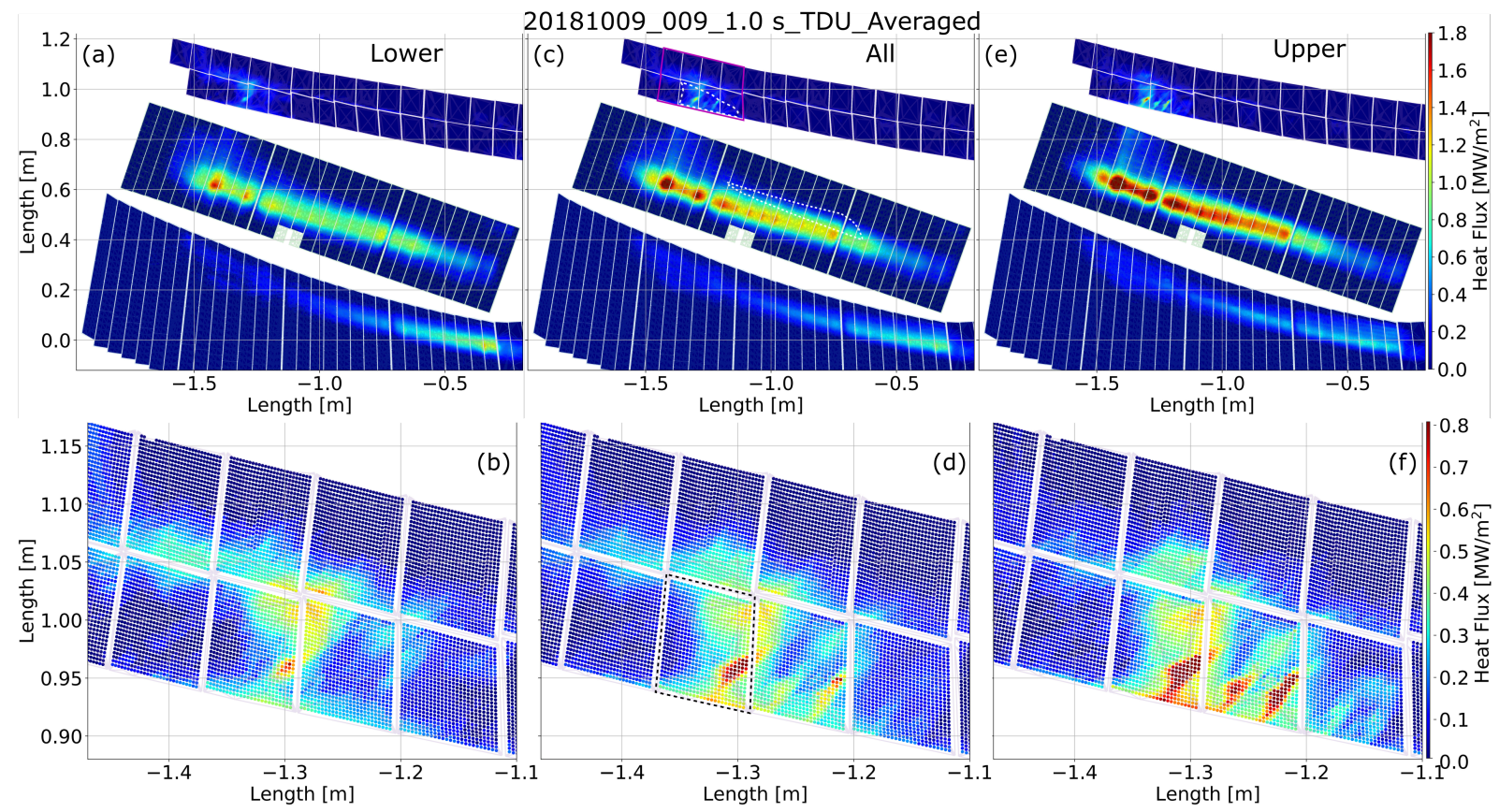

Figure 5: Heat flux maps resulting from averages among divertor modules (1-4). The sub-figures (a), (c), (e) show an overview of the heat flux patterns on the low-iota part of the TDU including the vertical target, a part of the horizontal target and the baffle plate, while (b), (d), (f) are zoom-in sub-figures focusing on the region with the 8 loaded baffle tiles (surrounded by a purple line in (c)) with different scale of the colorbar. (a), (b) are averaged results for the lower TDUs, (c), (d) for all-TDUs and (e), (f) for the upper TDUs. In (d), the most critically loaded baffle tile is marked with a black dashed line as in figure 1 . The areas in (c) marked by the white-dashed line are sharing the same flux tube. Here, they are mapped to the same module, but in reality they connect neighbouring modules.
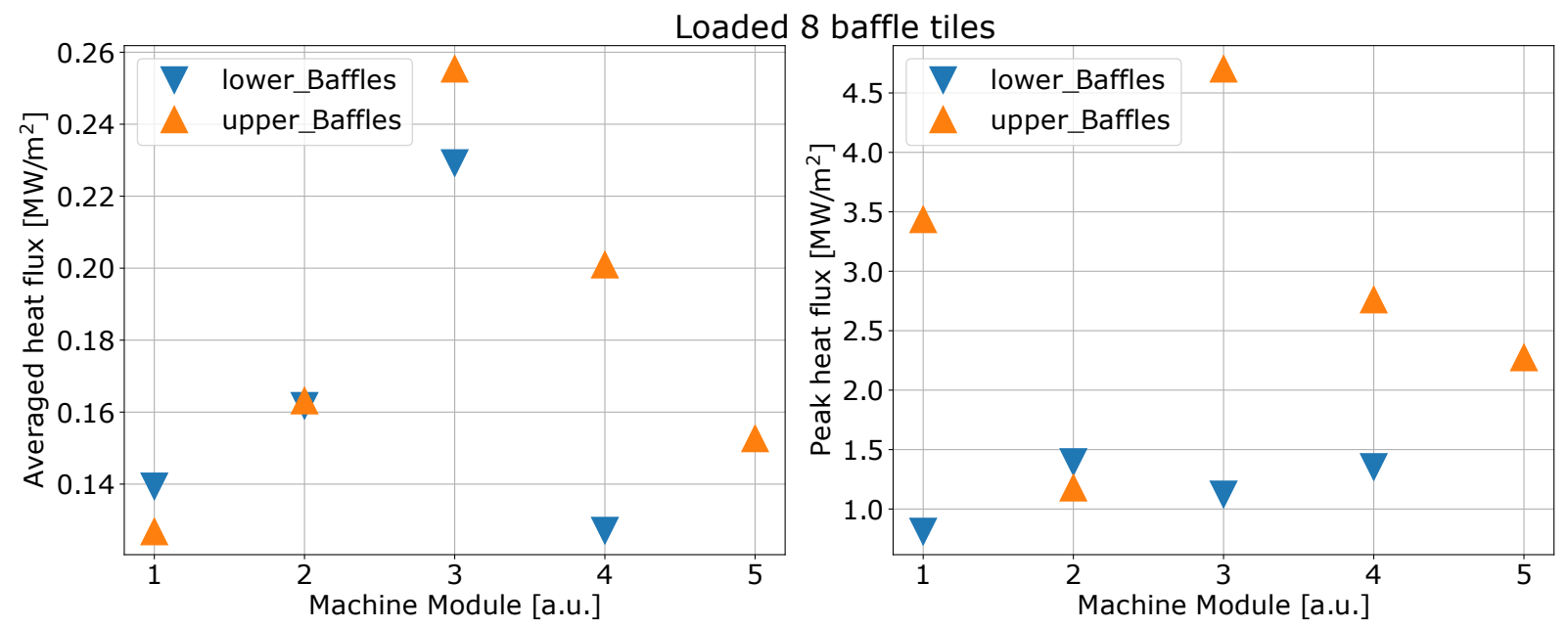

Figure 6: Average and peak heat flux of the 8 baffle tiles (surrounded by a purple line in figure 5) for each TDU). 


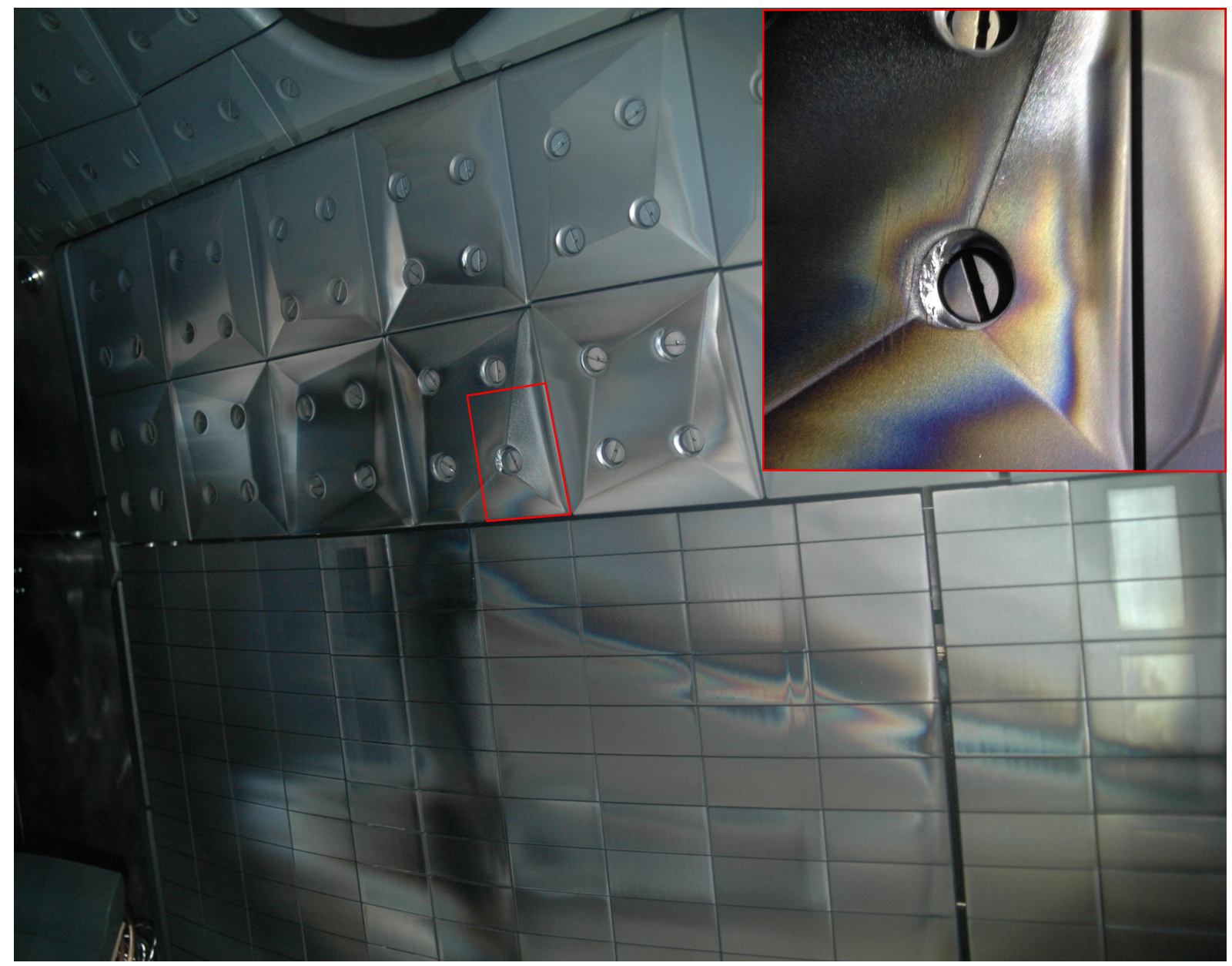

Figure 7: Pictures taken during the in-vessel inspection showing the eroded chamfer of the screw in $5 \mathrm{u}$, as well as the multiple scratches nearby. The screw region is marked with a red quadrangle and a zoom-in figure is embedded at the top right.

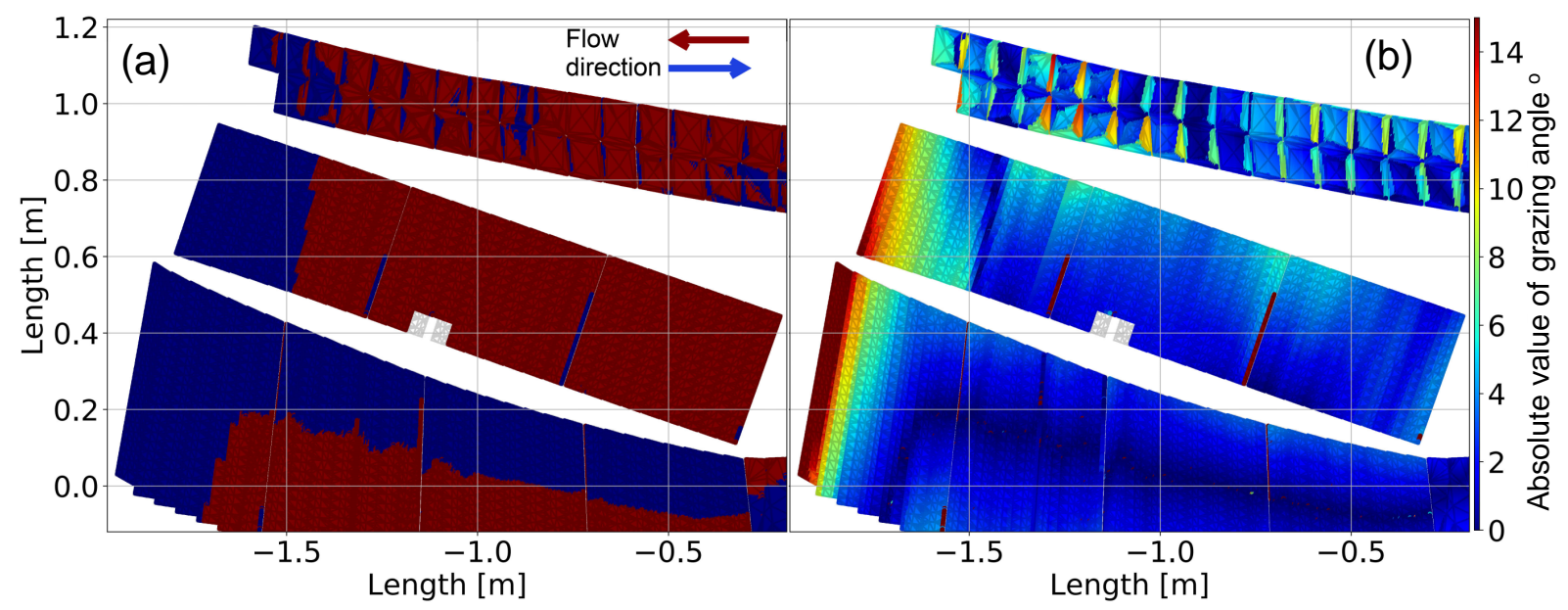

Figure 8: (a) Plasma flow directions to different components simulated for the high-mirror configuration. The watershed is located at the intersection between the red (flow towards low-iota end) and blue (flow from low-iota end) regions. (b) Absolute value of the grazing angles of field lines hitting the components in the high-mirror configuration. 

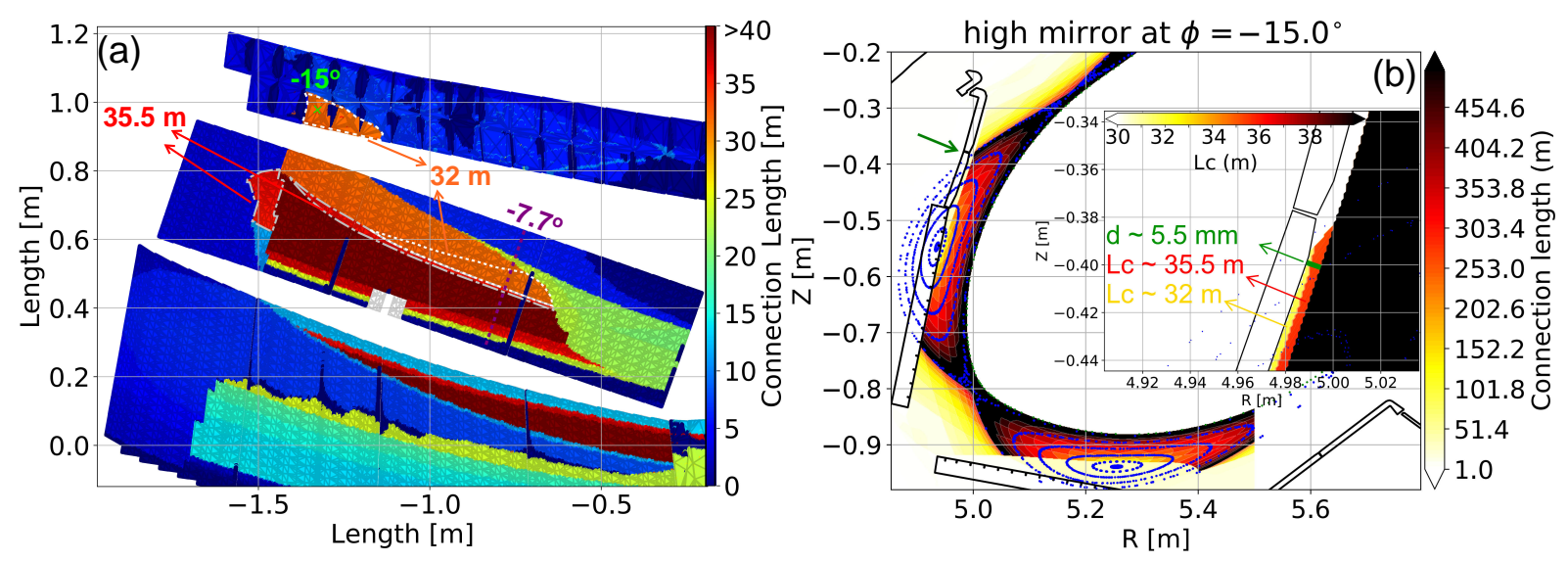

Figure 9: (a) Distribution of $L_{\mathrm{c}}$ for high-mirror configuration, mapped to the identical 2D plane as shown for the experimental results (figure 5). The area marked with the white dashed line is stellarator-symmetrically mapped region with $L_{\mathrm{c}}=32 \mathrm{~m}$ connecting the baffle plate and the vertical target. Areas marked with grey dash-dotted lines share the flux tube with $L_{\mathrm{c}}=35.5 \mathrm{~m}$. (b) $L_{\mathrm{c}}$-plot at toroidal angle $-15^{\circ}$ (the center of the most critical baffle tile whose location is marked with a green cross in (a)) in the high-mirror configuration with an enlarged figure showing a distance of $\sim 5.5 \mathrm{~mm}$ between the critical baffle tile and the hot plasma flowing along the separatrix.

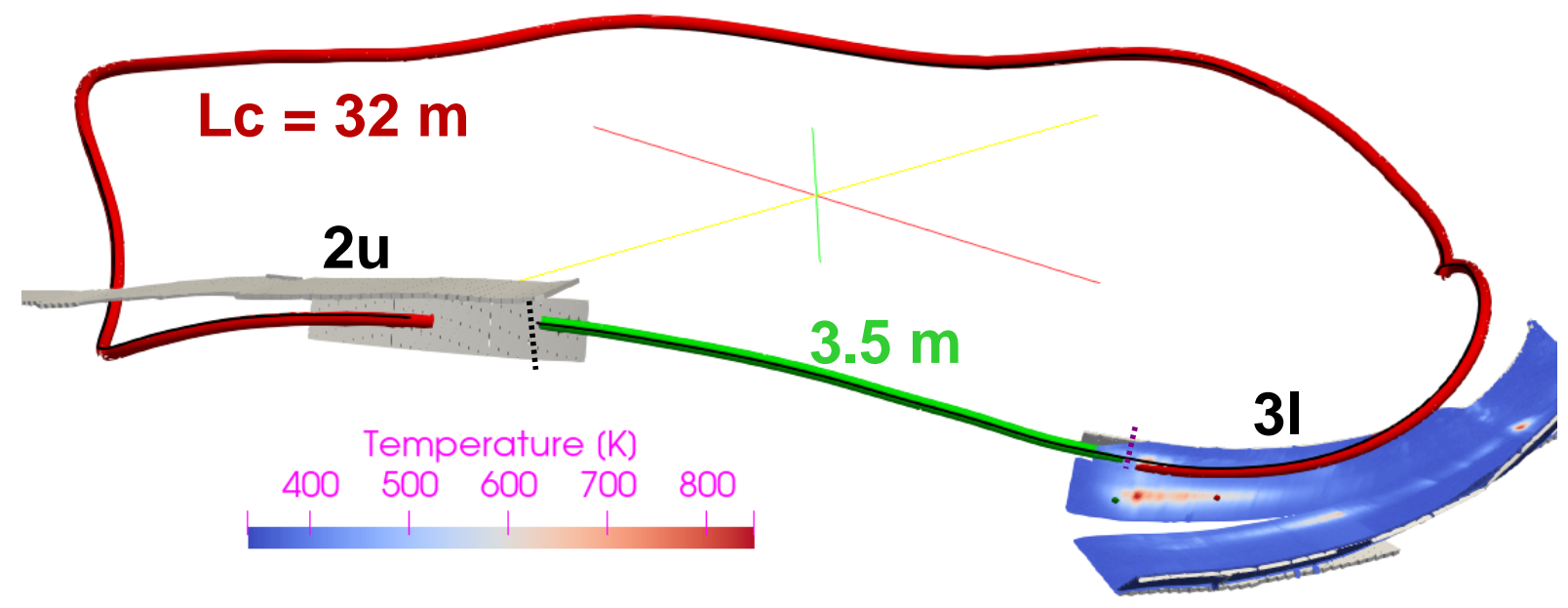

Figure 10: Field-line traces showing the connections between the loaded baffle tiles and the vertical target $\left(L_{c}=32 \mathrm{~m}\right.$ in red, $3.5 \mathrm{~m}$ in green). The $35.5 \mathrm{~m}$ long field line is in black. The experimental temperature measurement shown in figure 4 is rendered to the components. The end points of field lines at $2 u$ are stellarator-symmetrically mapped to 31 represented with dots with respective colors. The watershed on $2 \mathrm{u}$ and 31 are indicated with dashed lines for the vertical target (black) and for the baffle plate (purple). 


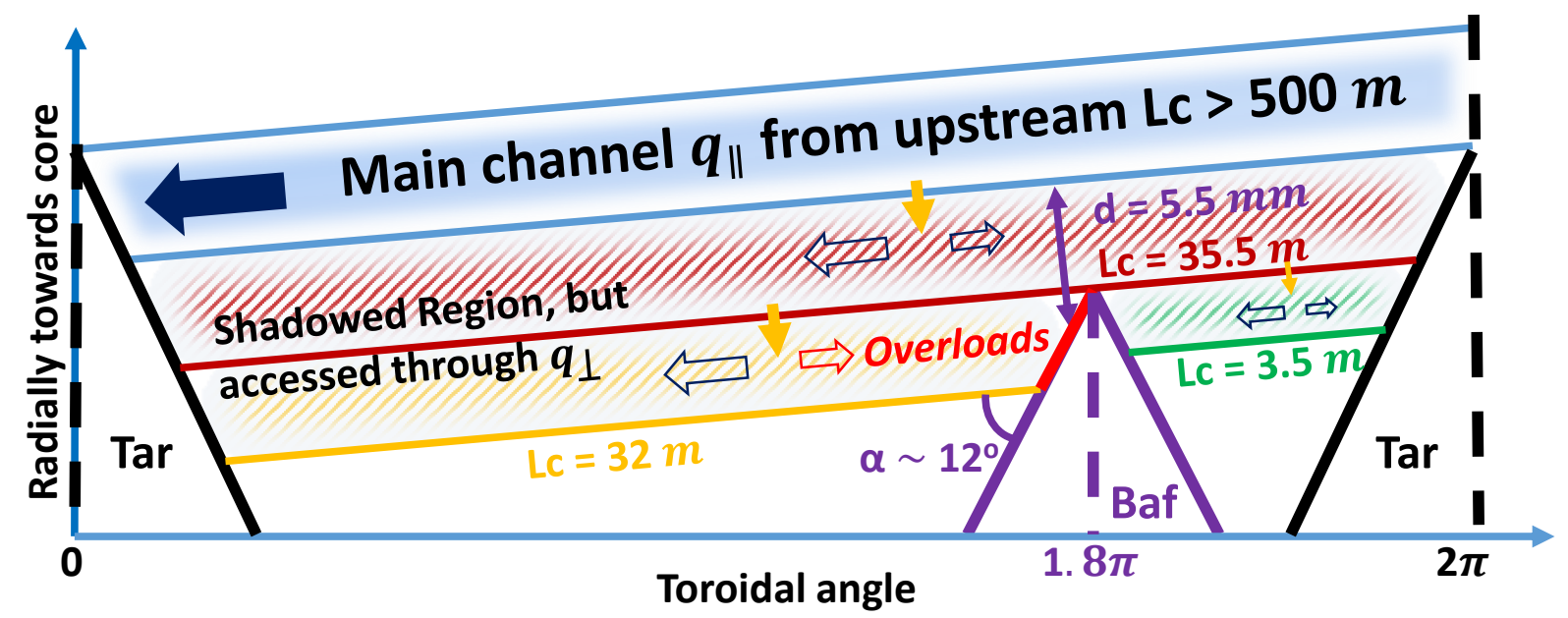

Figure 11: Schematic sketch showing the heat transport causing the baffle overloads.

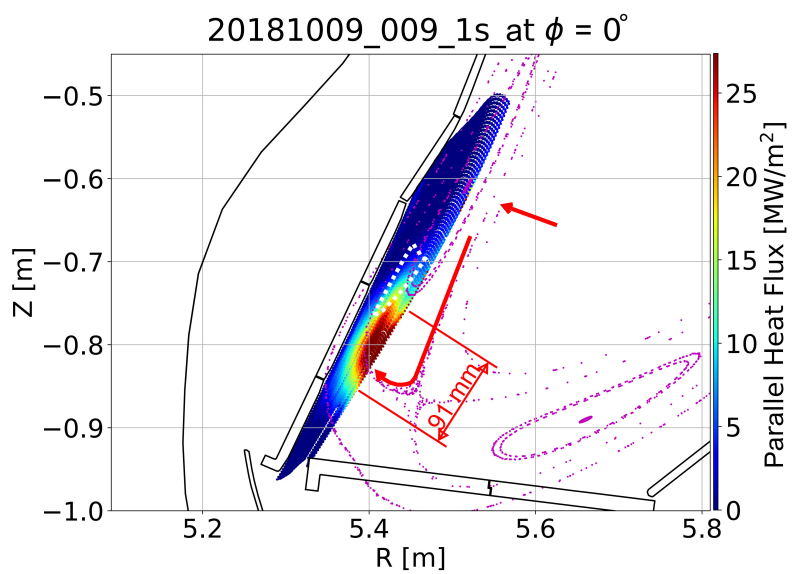

Figure 12: $q_{\|}$(averaged over all TDUs) to the vertical target for discharge 20181009_009 at $1 \mathrm{~s}$ traced backwards to a poloidal cross-section at $0^{\circ}$ (location seen in figure 1 ). The area marked with the white-dotted line indicates the $32 \mathrm{~m}$ flux tube region connecting to the baffle plate. 

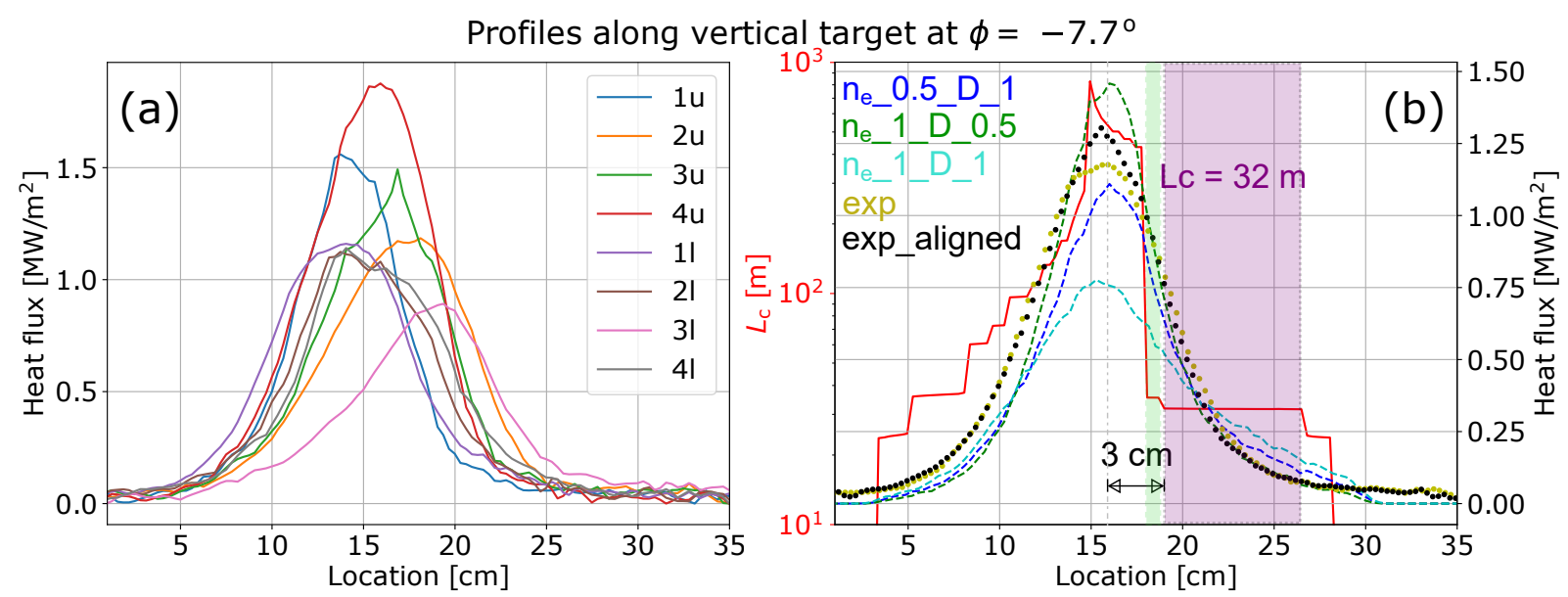

Figure 13: (a) Heat flux profiles from different machine modules at correspondent identical locations along the vertical target for discharge 20181009_009 at 1s. The line location is indicated as dashed purple line in figure 9 (a), which is for 11 at a toroidal angle of $-7.7^{\circ}$. Location 0 is defined as the pumping gap end (close to the horizontal target) of the vertical target. (b) Experimentally determined average heat flux using different averaging methods (black dots for peak aligned, yellow dots for location fixed) and $L_{\mathrm{c}}$ profile (red solid line in logarithmic scale). Dashed lines are EMC3-EIRENE simulations using a heating power of $3 \mathrm{MW}$, and a perpendicular heat transport coefficient of $\chi=3 D$, and three combinations of different separatrix electron densities $\left(n_{e}\right.$ in $\left.10^{19} \mathrm{~m}^{-3}\right)$ and anomalous particle diffusivity $\left(D\right.$ in $\left.\mathrm{m}^{2} \mathrm{~s}^{-1}\right)$. The location of the flux tubes with $L_{\mathrm{c}}=35.5 \mathrm{~m}$ and $32 \mathrm{~m}$ are marked with green and purple vertical stripes.

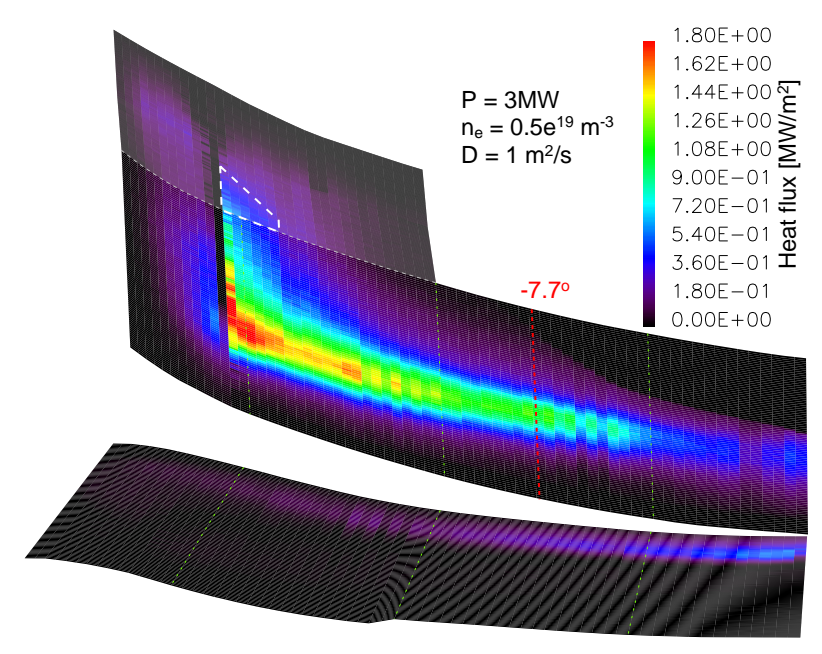

Figure 14: EMC3-EIRENE simulation reproducing the baffle loads. 


\section{References}

[1] T. S. Pedersen, R. König, M. Krychowiak et al. First results from divertor operation in Wendelstein 7-X. Plasma Physics and Controlled Fusion, 61 (1), 014035 (2018).

[2] T. Klinger, T. Andreeva, S. Bozhenkov et al. Overview of first Wendelstein 7-X high-performance operation. Nuclear Fusion, 59 (11), 112004 (2019).

[3] R. C. Wolf, A. Alonso, S. Äkäslompolo et al. Performance of Wendelstein 7-X stellarator plasmas during the first divertor operation phase. Physics of Plasmas, 26 (8), 082504 (2019).

[4] T. S. Pedersen, R. König, M. Jakubowski et al. First divertor physics studies in Wendelstein 7-X. Nuclear Fusion, 59 (9), 096014 (2019).

[5] J. Geiger, C. D. Beidler, Y. Feng et al. Physics in the magnetic configuration space of W7-X. Plasma Physics and Controlled Fusion, 57 (1), 014004 (2014).

[6] S. Äkäslompolo, M. Drevlak, Y. Turkin et al. Modelling of NBI ion wall loads in the W7-X stellarator. Nuclear Fusion, 58 (8), 082010 (2018).

[7] Y. Feng, M. Kobayashi, T. Lunt et al. Comparison between stellarator and tokamak divertor transport. Plasma Physics and Controlled Fusion, 53 (2), 024009 (2011).

[8] D. Zhang, R. König, Y. Feng et al. First Observation of a Stable Highly Dissipative Divertor Plasma Regime on the Wendelstein 7-X Stellarator. Phys. Rev. Lett., 123, 025002 (2019).

[9] Y. Gao, M. W. Jakubowski, P. Drewelow et al. Methods for quantitative study of divertor heat loads on W7-X. Nuclear Fusion, 59 (6), 066007 (2019).

[10] M. Jakubowski, P. Drewelow, J. Fellinger et al. Infrared imaging systems for wall protection in the W7-X stellarator (invited). Review of Scientific Instruments, 89 (10), $10 \mathrm{E} 116$ (2018).

[11] A. Puig Sitjes, M. Jakubowski, A. Ali et al. Wendelstein 7-X Near Real-Time Image Diagnostic System for Plasma-Facing Components Protection. Fusion Science and Technology, 74 (1-2), 116 (2018).

[12] F. Pisano, B. Cannas, M. W. Jakubowski et al. Towards a new image processing system at Wendelstein 7-X: From spatial calibration to characterization of thermal events. Review of Scientific Instruments, 89 (12), 123503 (2018).

[13] T. Andreeva, T. Bräuer, M. Endler et al. Analysis of the Magnetic Field Perturbations during the Assembly of Wendelstein 7-X. Fusion Science and Technology, 46 (2), 388 (2004).

[14] M. Otte, D. Aßmus, C. Biedermann et al. Setup and initial results from the magnetic flux surface diagnostics at Wendelstein 7-X. Plasma Physics and Controlled Fusion, 58 (6), 064003 (2016).

[15] S. Bozhenkov, M. Otte, C. Biedermann et al. Measurements and correction of the 1/1 error field in Wendelstein 7-X. Nuclear Fusion, 59 (2), 026004 (2018). 
[16] S. A. Lazerson, S. Bozhenkov, B. Israeli et al. Error fields in the Wendelstein 7-X stellarator. Plasma Physics and Controlled Fusion, 60 (12), 124002 (2018).

[17] S. A. Lazerson, Y. Gao, K. Hammond et al. Tuning of the rotational transform in Wendelstein 7-X. Nuclear Fusion, 59 (12), 126004 (2019).

[18] K. C. Hammond, Y. Gao, M. Jakubowski et al. Drift effects on W7-X divertor heat and particle fluxes. Plasma Physics and Controlled Fusion, 61 (12), 125001 (2019).

[19] S. Bozhenkov, J. Geiger, M. Grahl et al. Service oriented architecture for scientific analysis at W7-X. An example of a field line tracer. Fusion Engineering and Design, 88 (11), 2997 (2013).

[20] Y. Feng, F. Sardei, J. Kisslinger et al. 3D Edge Modeling and Island Divertor Physics. Contributions to Plasma Physics, 44 (1-3), 57 (2004).

[21] D. Reiter, M. Baelmans and P. Börner. The EIRENE and B2-EIRENE Codes. Fusion Science and Technology, 47 (2), 172 (2005).

[22] M. Jakubowski, A. Ali, P. Drewelow et al. 3D heat and particle fluxes in Wendelstein 7-X. 27th IAEA Fusion Energy Conference (2018).

[23] F. Effenberg, H. Niemann, Y. Feng et al. Investigation of 3D effects on heat fluxes in performance-optimized island divertor configurations at Wendelstein 7-X. Nuclear Materials and Energy, 18, 262 (2019).

[24] Y. Gao, J. Geiger, M. W. Jakubowski et al. Effects of toroidal plasma current on divertor power depositions on Wendelstein 7-X. Nuclear Fusion, 59 (10), 106015 (2019).

[25] A. P. Sitjes, Y. Gao, M. Jakubowski et al. Observation of thermal events on the plasma facing components of Wendelstein 7-X. Journal of Instrumentation, 14 (11), C11002 (2019).

[26] H. Greuner, B. Böswirth, J. Boscary et al. Final design of W7-X divertor plasma facing components - tests and thermo-mechanical analysis of baffle prototypes. Fusion Engineering and Design, 66-68, 447 (2003). 22nd Symposium on Fusion Technology.

[27] K. Brunner, T. Akiyama, M. Hirsch et al. Real-time dispersion interferometry for density feedback in fusion devices. Journal of Instrumentation, 13 (09), P09002 (2018).

[28] A. Herrmann, W. Junker, K. Gunther et al. Energy flux to the ASDEX-Upgrade diverter plates determined by thermography and calorimetry. Plasma Physics and Controlled Fusion, 37 (1), 17 (1995).

[29] D. Rudakov, C. Chrobak, R. Doerner et al. Arcing and its role in PFC erosion and dust production in DIII-D. Journal of Nuclear Materials, 438, S805 (2013). Proceedings of the 20th International Conference on Plasma-Surface Interactions in Controlled Fusion Devices.

[30] S. Zoletnik, G. Anda, C. Biedermann et al. Multi-diagnostic analysis of plasma filaments in the island divertor. Plasma Physics and Controlled Fusion, 62 (1), 014017 (2019). 
[31] V. Perseo, F. Effenberg, D. Gradic et al. Direct measurements of counter-streaming flows in a low-shear stellarator magnetic island topology. Nuclear Fusion, 59 (12), 124003 (2019).

[32] Y. Feng, F. Sardei, P. Grigull et al. Physics of island divertors as highlighted by the example of W7-AS. Nuclear Fusion, 46 (8), 807 (2006).

[33] P. Grigull, K. McCormick, J. Baldzuhn et al. First island divertor experiments on the W7-AS stellarator. Plasma Physics and Controlled Fusion, 43 (12A), A175 (2001).

[34] M. Endler, J. Fellinger, H. Hölbe et al. Managing leading edges during assembly of the Wendelstein 7-X divertor. Plasma Physics and Controlled Fusion, 61 (2), 025004 (2018).

[35] K. U. Riemann. The Bohm criterion and sheath formation. Journal of Physics D: Applied Physics, 24 (4), 493 (1991).

[36] G. Wurden, C. Biedermann, F. Effenberg et al. Limiter observations during W7-X first plasmas. Nuclear Fusion, 57 (5), 056036 (2017).

[37] P. Stangeby. The Plasma Boundary of Magnetic Fusion Devices. Nicki Dennis, Institute of Physics Publishing, Bristol (2000).

[38] A. Loarte, S. Bosch, A. Chankin et al. Multi-machine scaling of the divertor peak heat flux and width for L-mode and H-mode discharges. Journal of nuclear materials, 266, 587 (1999). 